\title{
Open Access im rechtswissenschaftlichen Verlag
}

Johannes Rux"

A. Differenzierung tut not ............. 70

I. Open Access für Praktikerliteratur:

Kommentare, Handbücher und

Fachzeitschriften

II. Open Access für wissenschaftliche

Monografien, Sammelbände und

Zeitschriften

B. Open Access und Wissenschaftskommunikation

C. Ein Fallbeispiel: Open Access bei

Nomos

I. Verlagsrichtlinien für die Veröffent-

lichung bei Nomos
II. Open Access für Zeitschriften ....... 78

III. Open Access für Bücher ............ 80

1. Kosten und Finanzierung ........ 80

2. Modell 1: Open Access mit paralleler Druckausgabe (hybrides Modell)

3. Modell 2: Ausschließliche Online-Veröffentlichung mit Open Access (eOnly-Modell) .... 83

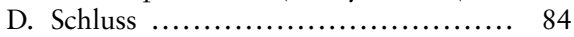

In der Diskussion über Open Access ist oft von „den Wissenschaftsverlagen“ die Rede, die teilweise in keinem guten Licht erscheinen. Tatsächlich erfüllen professionelle Wissenschaftsverlage eine wichtige Aufgabe im System der Wissenschaft, indem sie zur Qualitätssicherung beitragen und gemeinsam mit den Bibliotheken für eine möglichst effiziente Verbreitung wissenschaftlicher Erkenntnisse sorgen. Dies gilt auch und insbesondere für elektronische Publikationen, die weit über die Veröffentlichung der eigentlichen Texte hinaus gehen muss und zwar unabhängig davon, ob der Zugang frei oder hinter einer Bezahlschranke erfolgt. Bei Open Access müssen aber neue Wege gefunden werden, um den mit der Publikation verbundenen Aufwand abzudecken. Der folgende Beitrag soll zeigen, dass nachhaltige Open Access Geschäftsmodelle möglich sind.

\section{A. Differenzierung tut not}

In der Diskussion über Open Access in der (Rechts-)Wissenschaft ist oft von „den Verlagen“ die Rede. Während in den Naturwissenschaften, der Medizin und den Ingenieurwissenschaften (STM) wenige, weltweit tätige Großverlage dominieren, zeichnet sich die deutschsprachige Verlagsbranche in den Sozial- und Geisteswissenschaften im Allgemeinen und in der Rechtswissenschaft im Besonderen durch eine große Vielfalt und eine mittelständische Prägung aus. Jeder der einschlägigen Verlage bringt sein eigenes Verständnis seiner Stellung im System der Wissenschaft mit - das sich wiederum in ganz unterschiedlichen Geschäftsmodellen abbildet. Ich will mir nun nicht anmaßen, alle denkbaren Modelle darstellen zu können. Vielmehr kann ich nur für mein eigenes Haus sprechen, also für den Nomos Verlag.

* Apl. Prof. Dr. Johannes Rux lehrt Öffentliches Recht an der Eberhard-Karls-Universität Tübingen, ist Programmleiter Wissenschaft im Juristischen Lektorat des Nomos Verlags und Schriftleiter der „Rechtswissenschaft“. Dieser Beitrag geht auf eine Einladung der Herausgeber des Sonderbandes zurück. 
Die folgenden Ausführungen können möglicherweise weitgehend auf andere Wissenschaftsverlage übertragen werden, aber es muss jedem bewusst sein, dass es auch ganz andere Modelle gibt.

Bei der Diskussion über Open Access ist eine weitere Differenzierung notwendig: Auch in der Rechtswissenschaft gibt es „Grundlagenforschung“ die sich eher abstrakt mit dem Recht und seiner Anwendung auseinandersetzt, aber nicht den Anspruch erhebt, den RezipientInnen der Forschungsergebnisse konkrete Hinweise für die Lösung praktischer Probleme zu geben. Zwar ist die Unterscheidung zwischen Wissenschaft und Praxis nicht völlig trennscharf möglich. Es lassen sich aber zumindest grob (mindestens) zwei Bereiche unterscheiden: Kommentare, Handbücher und ein Teil der juristischen Zeitschriften haben in erster Linie die Bedürfnisse der PraktikerInnen in Justiz, Anwaltschaft, Verbänden und Wirtschaft im Blick, die für ihre berufliche Tätigkeit möglichst konkrete Antworten auf teilweise sehr spezielle Rechtsfragen suchen. Die Fachliteratur ist hier Teil einer Wertschöpfungskette, und es ist selbstverständlich, dass nicht nur die AutorInnen, sondern auch die Verlage, die solche Werke mit hohem Aufwand konzipieren und verfügbar machen, von den Erträgen profitieren, die die LeserInnen durch die Nutzung der Werke erlösen. Es erscheint daher angemessen und gerecht, dass der Zugriff auf die entsprechenden Werke für die Nutzer kostenpflichtig ist und dass die AutorInnen über Honorare an den Erlösen beteiligt werden. Auch ist nur so ist sichergestellt, dass diejenigen, die letzten Endes von der Nutzung der Werke materiell profitieren, die Kosten tragen, die mit der Entstehung dieser Werke verbunden sind. ${ }^{1}$

Dabei kann unterstellt werden, dass die Symptome der vielbeschworenen Zeitschriftenkrise ${ }^{2}$ in unserem Bereich, also bei juristischen Verlagen, nicht oder jedenfalls nicht in dem Maße zu beobachten sind, ${ }^{3}$ wie im STM-Bereich. Die Erlöse, die mit dem Verkauf von Praktikerwerken (Bücher und Zeitschriften) erzielt werden, sind daher (nur) kostendeckend. ${ }^{4}$

1 Hier unterscheidet sich die Publikationslandschaft m.E. auch grundlegend von der im STM-Bereich, in dem die meisten Publikationen „grundlagenbezogen“ erscheinen. Erkenntnisse, die unmittelbar praktisch nutzbar wären, werden in Regel nicht im Detail veröffentlicht, schon gar nicht Open Access, sondern in reduzierter Form in Patentschriften und anderen Instrumenten zum Schutz des geistigen Eigentums.

2 Massive Preissteigerungen von Zeitschriften-Abonnements, verbunden mit einer in den Geschäftsberichten der Verlage ausgewiesenen Rendite, die unanständig wirkt.

3 Es gibt auch bei juristischen Zeitschriften Preissteigerungen, die aber in erster Linie auf Rückgänge bei den Abonnements zurückzuführen sind. Dabei sind diese Rückgänge weniger Indiz für mangelnde oder sinkende Attraktivität der Zeitschriften, als Folge des Umstandes, dass Einzel-Abonnements zunehmend durch Institutionelle Abonnements verdrängt werden, mit denen allen NutzerInnen der jeweiligen Einrichtung der parallele Zugang zur jeweiligen Zeitschrift einschließlich des Archives eröffnet wird. Für Bücher ist die Lage vergleichbar: Wo Bibliotheken in früheren Zeiten Mehrfachexemplare angeschafft haben, wird heute oft eine Lizenz zur unbeschränkten gleichzeitigen Nutzung des Werkes vereinbart.

4 Wobei zu den Kosten auch ein angemessener Gewinn für die Eigentümer des Verlags gehört. 


\section{Open Access für Praktikerliteratur: Kommentare, Handbücher und Fachzeitschriften}

Open Access erscheint für juristische Praktikerliteratur keine sinnvolle Option zu sein: Würden die bisherigen Erlöse der Verlage aus dem Verkauf von Büchern und Zeitschriften-Abonnements durch kostendeckende Open Access-Gebühren ersetzt, würde das zum einen im Ergebnis zu einer massiven Subventionierung derjenigen NutzerInnen führen, die z.B. als Anwälte mit der Nutzung der Werke (viel) Geld verdienen. Zum anderen würden die AutorInnen von einer Beteiligung an den Erlösen ausgeschlossen, die Dritte durch die Verwertung ihrer Forschungsergebnisse erzielen. Die Verschiebungen wären noch gravierender, wenn man unterstellt, dass die Open Access-Gebühren jedenfalls zu einem überwiegenden Anteil von der öffentlichen Hand getragen werden müssten.

Wenig hilfreich sind auch Vorschläge, diejenigen AutorInnen, die hauptberuflich an Hochschulen und oder Forschungseinrichtungen tätig sind und so ihren Lebensunterhalt verdienen, dazu zu verpflichten, ihre Forschungsergebnisse immer open access zu veröffentlichen. Unabhängig von dem offensichtliche Konflikt mit der Wissenschaftsfreiheit, die auch das Recht umfasst, frei darüber zu entscheiden, ob, wo und wie die eigenen Erkenntnisse publiziert werden, ${ }^{5}$ ist das ein Problem, das im Rahmen des Nebentätigkeitsrechts geklärt werden muss - insbesondere dann, wenn die Betroffenen für ihre Tätigkeit als AutorIn Ressourcen des Arbeitgebers nutzen und damit Einkünfte in Form von Autorenhonoraren generieren. ${ }^{6}$

\section{Open Access für wissenschaftliche Monografien, Sammelbände und Zeitschriften}

Ganz anders als bei der „Praktikerliteratur“ stellt sich die Lage bei der „Grundlagenforschung" dar, also bei denjenigen Werken, in denen WissenschaftlerInnen ihre Erkenntnisse publizieren, die nicht auf konkrete Rechtsfragen im Zusammenhang mit ganz spezifischen Rechtsproblemen bezogen sind. Typischerweise handelt es sich hier um wissenschaftliche Monografien, Sammelbände und theorielastige Zeitschriften, die derzeit vor allem von Bibliotheken abonniert werden. Ob, wann und in welchem Zusammenhang die hier veröffentlichten Erkenntnisse praktisch bedeutsam werden, ist zum Zeitpunkt der Veröffentlichung in der Regel nicht absehbar.

Der Aufwand der Verlage scheint bei diesen Werken oft geringer zu sein als bei den Praktikerwerken. Die Texte werden in der Regel fertig geliefert, und jedenfalls bei Qualifikationsschriften ist ein intensives inhaltliches Lektorat ausgeschlossen, da die Arbeiten in der Regel in der Fassung veröffentlicht werden müssen, in der sie

5 Vgl. dazu Dreier/Britz Art. 5 III GG (Wissenschaft), Rn. 26. Eine Entscheidung des BVerfG dazu steht noch aus. Fraglich ist auch die Regelungskompetenz der Landesgesetzgeber, vgl. den Vorlagebeschluss des VGH Mannheim vom 26.9.2017, 9 S 2056/16.

6 Das gilt im selben Maß für die Tätigkeit als GutachterIn. 
der Hochschule zur Begutachtung vorgelegt worden waren. ${ }^{7}$ Auch bei wissenschaftlichen Werken, die keine Qualifikationsschriften sind, haben Verlage in der Regel keine Möglichkeit für ein inhaltliches Lektorat, da es sich in der Regel um hoch spezialisierte Werke handelt, die nur von ausgewiesenen Fachleuten in dem betreffenden Gebiet überhaupt verstanden, geprüft und „verbessert“ werden könnten.

Ein Sprachlektorat erscheint in Zeiten der automatisierten Rechtschreibprüfung obsolet. Die Erfahrungen deuten darauf hin, dass die automatisieren Verfahren ähnlich genau sind, wie die manuelle Korrektur. Anders sieht es bei einem Fremdsprachenkorrektorat aus, das durch MuttersprachlerInnen erfolgen sollte. Dies ist - je nach Sprache - sehr aufwändig ${ }^{8}$ und kostenintensiv ${ }^{9}$ und muss ggf. gesondert angeboten und abgerechnet werden.

Um festzustellen, ob eine Arbeit überhaupt für die Veröffentlichung im Verlag geeignet ist, können Verlage zwar auf die Ergebnisse der Begutachtung in akademischen Verfahren zurückgreifen. Im Übrigen bleibt ihnen zunächst nur eine Evidenzkontrolle, ob eine Arbeit formal wissenschaftlichen Anforderungen genügt. Aber und diesem Umstand kommt immer größere Bedeutung zu - sie können und müssen die Qualität der eingereichten Manuskripte darüber hinaus durch die Einbeziehung von Reihen- und Zeitschriftenherausgebern ${ }^{10}$ und ggf. von externen GutachterInnen prüfen und auf diesem Wege Anregungen oder Auflagen für die Überarbeitung formulieren. ${ }^{11}$ Diese Qualitätskontrolle ist mit erheblichem Aufwand ver-

7 Dies ist durchaus konsequent, wenn man davon ausgeht, dass es sich um „Grundlagenforschung“ handeln soll. Die Lage unterscheidet sich jedenfalls grundlegend von der im angelsächsischen Sprachraum, wo kaum eine Qualifikationsschrift in der ursprünglichen Fassung publiziert wird. Vielmehr überarbeiten die AutorInnen ihre Werke mit Unterstützung der Verlage aufwendig, so dass sie auch für Praktiker attraktiv werden. Tatsächlich nehmen selbst wissenschaftliche Bibliotheken Werke nicht in den Bestand auf, wenn diese als „thesis“ erkennbar sind. Nur durch die an den Bedürfnissen der Praxis ausgerichtete Überarbeitung können die Verlage auf die Absatzzahlen kommen, die sie für die Deckung ihrer Kosten benötigen. Das führt auf der einen Seite dazu, dass die AutorInnen nicht durch Publikationskostenbeihilfen an der Finanzierung der Veröffentlichung beteiligt werden müssen. Auf der anderen Seite hängt die Publikation von der unmittelbaren Verwertbarkeit der Texte ab, so dass Erkenntnisse, deren Verwertbarkeit noch nicht erkennbar sind, unter den Tisch zu fallen drohen.

8 Erfahrene KorrektorInnen können ca. 10-12 Druckseiten pro Stunde gründlich lesen.

9 Es kommt durchaus darauf an, ob die KorrektorInnen in einem Hochlohnland leben. Für Korrekturen englischsprachiger Texte kann man hingegen durchaus auf Länder mit einem deutlich niedrigeren Gehaltsniveau ausweichen.

10 Es mutet merkwürdig an, wenn etwa der Schweizerische Nationalfonds (SNF) die Begutachtung durch Reihenherausgeber nicht als Qualitätsnachweis akzeptiert. Dies spiegelt ein unbegründetes Vorurteil wider, nach dem sich die Herausgeber einer Reihe in erster Linie von dem Bedürfnis leiten lassen, die eigene Reihe zu „befüllen“ - obwohl sie damit ihren eigenen Ruf untergraben würden.

11 Dazu gleich mehr (B.). 
bunden und setzt eine hohe allgemeine Fachkompetenz ${ }^{12}$ des Lektorats und seine enge Verzahnung mit der Wissenschaft voraus.

Abnehmer solcher Werke sind heutzutage in erster Linie Bibliotheken, die es (noch) als ihre Aufgabe ansehen, wissenschaftliche Werke systematisch zu sammeln, um ihren NutzerInnen den Zugang zu den Forschungsergebnissen zu eröffnen. Ob dieses Angebot wahrgenommen wird, ist zum Zeitpunkt des Erwerbs völlig unabsehbar. ${ }^{13}$ Die Leistung des Verlags besteht hier darin, das Werk selbst so aufzubereiten und durch Metadaten zu ergänzen, dass es möglichst leicht gefunden werden kann - und zwar nicht nur im OPAC der Bibliothek und in einer vom Verlag eingerichteten Datenbank, sondern in einer beliebigen Vielzahl von Datenbanken, Suchmaschinen, Repositorien etc. Dafür müssen entsprechende Schnittstellen eingerichtet werden. Fast noch wichtiger ist die Anreicherung um (mehrsprachige) Abstracts, ${ }^{14}$ Schlagworte, ${ }^{15}$ Klassifikationen ${ }^{16}$ und andere Metadaten, die dazu beitragen, die Inhalte möglichst weit und effizient zu verbreiten.

Die Lage unterscheidet sich damit grundlegend von der Situation bei der Praktikerliteratur: Hier muss der Verlag in erster Linie potenzielle LeserInnen erreichen, die bereit sind, für die Literatur Geld auszugeben. Dafür ist es in der Regel gut, wenn die LeserInnen in ein „geschlossenes“ System geführt werden, in dem sie möglichst alles finden, was sie für ihre Tätigkeit benötigen. Bei der Grundlagenforschung oder der Wissenschaftsliteratur im engeren Sinne sind hingegen die AutorInnen die wichtigste Zielgruppe. Die Verlage müssen alles daransetzen, dass die Werke ihrer AutorInnen wahrgenommen und zitiert werden. Das setzt wiederum voraus, dass diese Werke möglichst einfach zugänglich sind und dauerhaft verfügbar bleiben müssen. Es kommt dabei nicht darauf an, auf welchem Wege der Zugriff erfolgt, entscheidend ist, dass er überhaupt erfolgt.

12 Gemeint ist die Fähigkeit, die wissenschaftliche Qualität von Texten beurteilen zu können. Das setzt viel Erfahrung voraus, die oft - aber nicht immer - durch eigene wissenschaftliche Veröffentlichungen erworben wurde.

13 Es mutet daher merkwürdig an, wenn wissenschaftliche Bibliotheken bei der Bestellung von Datenbanken für wissenschaftliche Literatur immer häufiger auf die Nutzungszahlen aus der Vergangenheit abstellen (wohl auch deshalb, weil diese Daten nun erfasst werden können). Dabei unterscheiden die Bibliotheken oft nicht nach den Inhalten der verschiedenen Datenbanken: Natürlich wird die Nutzung einer Datenbank, die auch Lehrbücher und/oder Praktikerliteratur enthält, deutlich höher sein, als die Nutzung einer Datenbank, die gezielt wissenschaftliche Werke enthält, deren Bedeutung für den wissenschaftlichen Fortschritt oft erst lange nach der Veröffentlichung deutlich wird.

14 Über das Abstract bekommen potenzielle LeserInnen Hinweise auf die Relevanz des Werks für ihre eigenen Forschungen. Neben der Sprache, in der das Werk verfasst wurde, kommt englischen Texten Bedeutung zu, da die großen Zitations-Datenbanken, die Wissenschaftsliteratur auswerten, in der Regel nur auf die englischen Texte zugreifen.

15 Auch hier kommt der Mehrsprachigkeit Bedeutung zu, da potenzielle LeserInnen auf ein Werk aufmerksam gemacht werden können, das in einer Fremdsprache verfasst wurde.

16 Für den Verkauf kommt z.B. der so genannten THEMA-Klassifikation Bedeutung zu, die sich im Buchhandel international durchsetzt. In den Bibliotheken sind demgegenüber die verschiedensten Klassifikationssysteme im Einsatz. 
Damit wird deutlich, dass Wissenschaftsverlage integraler Bestandteil des Wissenschaftssystems sind. Zwar kochen wir auch nur mit Wasser, und ein/e engagierte/r und technisch gleichermaßen versierte/r AutorIn könnte viele der Aufgaben selbst übernehmen, die wir ihnen anbieten. Auch scheinen Universitätsverlage prima facie als sinnvolle Alternative. Ihr Leistungsspektrum bleibt aber in der Regel hinter dem Angebot professioneller Verlage zurück - oder sie können nur deshalb „kostengünstiger" publizieren, weil sie unentgeltlich Ressourcen der Hochschulen nutzen - die in der Regel anderswo sinnvoller eingesetzt werden könnten. Wie dem auch sei: Weil Wissenschaftsverlage unverzichtbare Dienstleistungen für die AutorInnen erbringen, ohne die entsprechenden Kosten durch Vertriebserlöse abdecken zu können, ist es im deutschen Sprachraum jedenfalls bei Büchern ${ }^{17}$ völlig üblich, dass sich die AutorInnen durch Beihilfen an den Kosten der Publikation beteiligen. ${ }^{18} \mathrm{Im}$ Ergebnis stellt sich bei Zeitschriften die Lage nicht anders dar, nur tragen hier jedenfalls heute nicht die AutorInnen die Kosten, sondern die HerausgeberInnen, die mit enormen persönlichen und in der Regel auch finanziellem Einsatz Internet-Projekte betrieben, die sich teilweise großer Anerkennung erfreuen - die Beispiele in diesem Heft illustrieren aber auch, dass diese Projekte vom Engagement einzelner Personen leben.

\section{B. Open Access und Wissenschaftskommunikation}

Open Access ist die konsequente und jedenfalls auf lange Sicht unverzichtbare Weiterentwicklung der klassischen Form der Wissenschaftskommunikation auch in den Geistes-, Sozial- und Rechtswissenschaften. Der Aktualitätsdruck mag nicht ganz so hoch sein, wie im STM-Bereich. Grundsätzlich besteht aber auch hier der Bedarf nach möglichst freiem Zugang zu Forschungsergebnissen, um auf diesen Ergebnissen aufzubauen und sie in Zusammenhängen zu nutzen, die mit dem ursprünglichen Gegenstand der Forschung nichts oder nur am Rande zu tun haben.

Ziel einer Transformation in Richtung Open Access sollte sein, die Kompetenz der bisherigen Partner des Wissenschaftssystems zu nutzen. Die Rolle der AutorInnen bleibt im Wesentlichen unverändert. Professionelle Verlage spielen eine wesentliche Rolle dabei, sicherzustellen, dass Open Access Publikationen denselben Qualitätskriterien genügen, die auch für gedruckte Veröffentlichungen gelten, und dass sie mindestens im gleichen Maße gut auffindbar und sichtbar sind. Sie müssen daher

17 Auch bei Zeitschriften sind Zuschüsse der Herausgeber oder einer am Thema interessierten Institution durchaus üblich. Häufig hängt die Existenz einer Zeitschrift aber auch davon ab, dass die Redaktionsarbeit durch Dritte finanziert wird, so dass nur ein Teil der gesamten Kosten über die Vertriebserlöse abgedeckt werden muss.

18 Ginge es nur um die Formalität der Veröffentlichung als Teil des Promotionsverfahrens, wäre das wohl deutlich günstiger zu haben. Tatsächlich gibt es Verlage, die auf Zuschüsse der AutorInnen verzichten, weil sie nur die Voraussetzung für die Aushändigung der Promotionsurkunde erfüllen und damit das Recht, den Doktortitel zu führen. Hier kommt der Eitelkeit der AutorInnen durchaus Bedeutung zu. Für einen ernst zu nehmenden Wissenschaftsverlag ist das jedoch keine Klientel. 
ihre Erfahrungen aus dem Verkaufsmarketing einbringen, aber auch neue Wege für die möglichst effiziente Verbreitung von Forschungsergebnissen finden. Die Bibliotheken werden ihre Rolle am stärksten verändern müssen, da sie sich nicht länger auf die Bevorratung von Inhalten für ihre NutzerInnen beschränken können. Dafür sind zentrale Datenspeicher sehr viel besser geeignet. Die Bibliotheken haben dann die Aufgabe, diese Speicher möglichst gut für ihre NutzerInnen zu erschließen. Das beginnt aber schon damit, dass sie den Angehörigen ihrer Einrichtung die Publikation ihrer Werke ermöglichen.

Die Bibliotheken haben diesen Transformationsprozess bereits begonnen, und zahlreiche Stellungnahmen aus Bibliotheken lassen hoffen, dass sie bereit sind, ihre Funktion neu zu definieren und z.B. Mittel, die bisher für den Bestandsaufbau genutzt wurden, für die Förderung von Open Access umzuwidmen. Die größte Herausforderung besteht hier wohl in der unabdingbaren Neuverteilung der Mittel, die den einzelnen Einrichtungen in Zukunft entsprechend dem Publikationsvolumen zugewiesen werden sollten. ${ }^{19}$

Es gibt durchaus Verlage, die bereit sind, diese Entwicklung aufzunehmen und entsprechende Geschäftsmodelle für nachhaltige Open Access-Modelle zu entwickeln. Nomos gehört dazu. Wir sprechen gerne mit Bibliotheken und Konsortien, wie wir gemeinsam die Transformation zu Open Access bewältigen können.

\section{Ein Fallbeispiel: Open Access bei Nomos}

Der Nomos Verlag ist sich der Herausforderungen bewusst, mit welchen sich die Verlagsbranche durch die Open Access-Bewegung konfrontiert sieht. Wir sehen jedoch auch - und das ist weit wichtiger - die Möglichkeiten, die Open Access für die Verbreitung aktueller Forschungsergebnisse bietet. Wir sind überzeugt, dass professionelle Verlage auch und gerade im Zeitalter des Open Access eine wichtige Rolle für die Gewährleistung der Qualität wissenschaftlicher Veröffentlichungen und die bestmögliche Verbreitung von Forschungsergebnissen in der Wissenschaft und in der Öffentlichkeit spielen.

Im Folgenden möchte ich unser aktuelles Modell für Open Access darstellen. Ich orientiere mich dabei an dem Inhalt unseres Internet-Angebotes für AutorInnen, habe aber versucht, allzu werbliche Aussagen zu vermeiden (auch wenn das Selbstlob natürlich berechtigt wäre).

19 Das setzt sich auch innerhalb der Einrichtungen fort, da bestimmte Fakultäten, Institute oder Einzelpersonen sehr viel mehr publizieren als andere und daher auch einen höheren Finanzierungsbedarf haben. 


\section{Verlagsrichtlinien für die Veröffentlichung bei Nomos}

Die folgenden Ausführungen gelten gleichermaßen für reguläre Veröffentlichungen, wie für Open Access-Publikationen. Die Qualität von Open Access-Publikationen darf selbstverständlich nicht hinter den Standards zurückbleiben, die wir für gedruckte Bücher und Zeitschriften anwenden. Der Umstand, dass ein/e AutorIn oder eine Institution für die Publikation eine Gebühr zahlen mag, darf keinen Einfluss auf die Annahme zur Veröffentlichung haben.

Im Nomos Verlag und seinen Imprints Academia und Ergon veröffentlichen wir mehr als 900 Werke jährlich, hinzu kommen etwa 70 Fachzeitschriften. Mehr als die Hälfte des Programms entfällt auf die Rechtswissenschaft, der Rest verteilt sich auf Sozial und Geisteswissenschaften. Das Programm reicht von der klassischen Wissenschaftsliteratur (ca. 400 juristische Monografien und Sammelbände) über Handbücher und Kommentare bis zur Studienliteratur. Als bisher einziger vorwiegend deutschsprachiger Buchverlag wurde Nomos seit 2017 nach Abschluss eines aufwendigen Begutachtungsverfahrens ${ }^{20}$ durch eine DFG-Allianzlizenz der Deutschen Forschungsgemeinschaft (DFG) gefördert. Man kann (und sollte) „Rankings" wissenschaftlicher Verlage mit Skepsis betrachten. ${ }^{21}$ Aber Nomos wird in solchen Rankings häufig als einer der ganz wenigen deutschsprachigen Verlage in die Spitzengruppen eingeordnet. ${ }^{22}$

Sämtliche Wissenschaftstitel durchlaufen ein strenges Begutachtungsverfahren. Dissertationen oder andere Qualifikationsarbeiten müssen mit überdurchschnittlich guten Noten bewertet worden sein, um bei Nomos veröffentlicht zu werden. ${ }^{23}$

20 In diesem Verfahren geht es einerseits um die Einhaltung definierter Lizenzierungsstandards, andererseits um ein angemessenes Preis-/Leistungsverhältnis. Im Mittelpunkt steht aber die fachliche Begutachtung der Inhalte.

21 Schließlich ist die Auswahl der maßgeblichen Faktoren im Ergebnis willkürlich. Es kommt zum einen auf das Fachgebiet, die Sprache der Veröffentlichungen, die Sprache der NutzerInnen. Teilweise werden explizit subjektive Wertungen genutzt, weil die scheinbar objektiven Ergebnisse bibliometrischer Systeme eben nicht alles auswerten - und nicht danach differenzieren, ob ein Werk zustimmend oder kritisch zitiert wird.

$22 \mathrm{Zu}$ erwähnen ist etwa das international renommierte SENSE Ranking (vgl. http://www.sense.nl/ gfx_content/documents/20170626_publishers_complete.xlsx - Link abgerufen am 8.8.2019.): Die Liste enthält 4.986 Einträge für 1.092 unterschiedliche Verlage (teilweise mit mehreren Standorten). 27 Einträge beziehen sich auf das Jahr 2017, Davon sind 15 Verlage (55,6\%) in den Stufen A und B. Nomos ist in Stufe B eingruppiert. Bezieht man auch die Bewertungen aus früheren Jahren ein, sind 114 der 1.092 Verlage $(10,4 \%)$ in den Stufen A und B. SENSE wird von der „Research School for Socio-Economic and

Natural Sciences of the Environment" an der Wageningen University (NL) verantwortet. Der Index wird aber auch von zahlreichen anderen Einrichtungen weltweit genutzt.

23 Nomos verlangt eine „überdurchschnittlich gute“ Bewertung. Das verlangt angesichts der je nach Hochschule unterschiedlichen Verteilung der Noten auf die einzelnen Stufen sehr gute Kenntnisse der einzelnen Fakultäten. Teilweise reicht selbst eine Bewertung mit „magna cum laude“ nicht sicher aus, weil deutlich mehr als die Hälfte der Arbeiten mit dieser Note oder besser bewertet wird. Teilweise kann ein „cum laude“ genügen, etwa wenn eine Fakultät eine zusätzliche Notenstufe (in der Regel „satis bene“) vergibt, um stärker differenzieren zu können und die spätere Erfahrung zeigt, dass „cum laude“ ein echtes Lob beinhaltet. 
Darüber hinaus erscheinen die allermeisten unserer Bücher in Schriftenreihen, die ihrerseits von renommierten WissenschaftlerInnen herausgegeben werden. Die HerausgeberInnen der Schriftenreihen sind für das Gesamtkonzept und die Struktur der Reihe verantwortlich und prüfen die eingereichten Manuskripte. Um sicherzustellen, dass die veröffentlichten Beiträge die geforderten Qualitätsstandards erreichen, können die ReihenherausgeberInnen die Beiträge an externe GutachterInnen weiterleiten, die in ihrem jeweiligen Fach führende ExpertInnen sind. Die HerausgeberInnen stellen sicher, dass die GutachterInnen unvoreingenommen und qualifiziert sind. ${ }^{24}$ Die endgültige Entscheidung über eine Veröffentlichung von Buchprojekten liegt bei unseren LektorInnen, die überwiegend selbst promoviert haben oder über eine vergleichbare akademische Qualifikation in ihrem jeweiligen Fachbereich verfügen.

Bei den Zeitschriften stellt sich die Lage zwar etwas anders dar. Im Ergebnis ist der Standard aber derselbe: Jede unserer Zeitschriften hat ein auf sie zugeschnittenes Begutachtungsverfahren. Die Bandbreite reicht von doppelt-blinden Peer-ReviewVerfahren (wie bei der „Rechtswissenschaft“) über die - im deutschen Sprachraum - klassische Begutachtung durch die HerausgeberInnen, die schließlich mit ihrem Namen für die Qualität der von ihnen herausgegebenen Zeitschrift bürgen, bis zu Mischverfahren, in denen neben oder anstelle der HerausgeberInnen Beiräte oder externe GutachterInnen in die Qualitätsprüfung einbezogen werden.

Zur Sicherstellung der Einhaltung der Regeln der guten wissenschaftlichen Praxis ist jeder Autor per Verlagsvertrag dazu verpflichtet, diese Regeln zu beachten. Grundsätzlich vertrauen wir allerdings darauf, dass sich die AutorInnen diese Pflicht zu eigen machen. Sollte es zu einem Plagiatsverdacht kommen, lehnen wir die betroffene Einreichung ab und informieren AutorInnen und HerausgeberInnen. Sollte ein Plagiat erst nach der Veröffentlichung entdeckt werden, wird der Vertrieb des Werks umgehend eingestellt, bis der Fall geklärt ist.

Wir schützen die Urheberrechte unserer AutorInnen. Das Recht zur Selbst-Archivierung für Beiträge in Zeitschriften und Sammelbänden, für die keine Honorare gezahlt werden, ist uns ebenso selbstverständlich, wie weit reichende Befugnisse zur Zweitverwertung.. ${ }^{25}$

\section{Open Access für Zeitschriften}

Nomos veröffentlicht derzeit zwei juristische Open Access Zeitschriften, die jeweils durch eine bestimmte Organisation finanziert werden:

24 Die GutachterInnen können selbstverständlich nicht nur die Annahmen oder Ablehnung eines Manuskriptes empfehlen, sondern auch Auflagen bzw. Anregungen formulieren und so zu einer Verbesserung der Texte beitragen.

25 Mehr dazu unter www.nomos.de/urheberrecht. 
- „Recht in Afrika/Law in Africa/Droit en Afrique“ (RIA) gemeinsam mit der Gesellschaft für afrikanisches Recht,

- „African Law Study Library“ (KAS ALSL), gemeinsam mit der Konrad Adenauer Stiftung.

Da die Adressaten dieser Zeitschriften größtenteils in Entwicklungsländern zuhause sind, deren Einrichtungen sich reguläre Zeitschriftenabonnements oft nicht leisten können, ist Open Access hier besonders gut geeignet, um die Inhalte optimal zu verbreiten. Die Zeitschriften werden vom Directory of Open Access Journals (DOAJ) gelistet. ${ }^{26}$

Jedes Jahr werden über 30 Fachartikel in diesen Zeitschriften veröffentlicht. Die Zeitschriften erscheinen unter einer CC-BY-NC-Lizenz. Diese Lizenz wurde in Abstimmung mit vielen Autoren gewählt, die eine unerlaubte kommerzielle Verwendung ihrer intellektuellen Arbeit verhindern möchten. ${ }^{27}$ Nomos ist jedoch auch bereit, Zeitschriften unter einer weniger restriktiven Lizenz zu verlegen, soweit es die Herausgeber und Autoren wünschen. Mit verschiedenen Zeitschriften laufen Gespräche über eine Transformation, die aber bisher daran scheitern, dass es noch keine tragfähigen Modelle zur dauerhaften Finanzierung der Publikationen gibt.

Während diese Zeitschriften den sogenannten "Goldenen Weg" des Open Access gehen, also nur elektronisch veröffentlicht werden und open access zugänglich sind, ermöglicht Nomos den Autorinnen und Autoren von Beiträgen in anderen Zeitschriften den "Grünen Weg" des Open Access, durch die so genannte SelbstArchivierung ihrer Beiträge. In Einzelfällen ermöglichen wir auch die OA-Publikation der elektronischen Fassung von Beiträgen. Grundsätzlich sehen wir das aber skeptisch und noch nicht als nachhaltigen Einstieg in eine Transformation zu Open Access: Je mehr Beiträge open access publiziert werden, desto geringer wird die Neigung ausfallen, ein bestehendes Abonnement zu halten. So lange „Article Processing Charges“ nur in Einzelfällen gezahlt werden und kein verlässliches Modell für die Finanzierung erkennbar ist, wäre ein Umstieg auf Open Access ein Weg ohne Umkehrmöglichkeit, weil die Abonnements bei einer Umstellung endgültig verloren wären.

26 Wobei die Aufnahme der KAS ALSL zum Zeitpunkt des Abschlusses dieses Manuskriptes noch im Begutachtungsverfahren hing. Da die zuständigen Gremien ehrenamtlich arbeiten, kann sich das Verfahren über längere Zeit hinziehen.

27 Im Gegensatz zu vielen Wissenschaftsorganisationen und Verfechtern möglichst unbeschränkte open acces (vgl, dazu Hamann, in diesem Heft S. 110) halten wir dieses Anliegen für legitim - vor allem in den Bereichen, in denen die Urheber keine Möglichkeit haben, ihre Erkenntnisse durch Patente zu schützen. 


\section{Open Access für Bücher}

Bis heute (August 2019) hat Nomos mehr als 160 juristische Monografien und Sammelbände unter einer CC-Lizenz veröffentlicht. Diese Zahl steigt stetig und es werden mehr und mehr Bücher unter einer CC-Lizenz parallel zur Druckausgabe veröffentlicht.

Die Veröffentlichung kann auch hier entweder im "Goldenen Weg" des Open Access erfolgen, bei dem die elektronische Fassung des Buches zeitgleich mit der gedruckten Fassung dieses Buches erfolgt. In anderen Fällen wird die elektronische Fassung nachträglich freigeschaltet ("Grüner Weg").

\section{Kosten und Finanzierung}

Die Publikation wissenschaftlicher Veröffentlichungen kostet Geld: Um sicherzustellen, dass ausgezeichnete Forschungsergebnisse diejenige Anerkennung erhalten, die sie verdienen, helfen wir WissenschaftlerInnen, ihre Arbeiten so aufzubereiten, dass sie in Katalogen, Indizes, Suchmaschinen und einer stets wachsenden Anzahl weiterer Services sichtbar sind. Es ist nicht damit getan, eine PDF-Datei auf einen Server hochzuladen und abzuwarten, bis der Google Crawler die Datei findet. Wir stellen mit großem Aufwand sicher, dass

- unsere wissenschaftlichen Bücher und Zeitschriften höchsten Qualitätsansprüchen gerecht werden,

- problemlos langfristig ${ }^{28}$ auf die Titel zugegriffen werden kann, ${ }^{29}$

- die Texte durch Metadaten, wie (mehrsprachige) Abstracts, Schlagworte ${ }^{30}$ und Klassifikationen ${ }^{31}$ ergänzt und dass diese Metadaten an die relevanten Reposito-

28 Wir nutzen dafür PORTICO und arbeiten an Projekten zur Langzeitarchivierung mit.

29 Derzeit werden noch oft PDF-Dokumente ausgegeben, die zum einen zitiersicher sind, zum anderen die für juristische Publikationen wichtige Möglichkeit bieten, die (oft kritischen) Fußnoten gemeinsam mit dem Haupttext zu lesen.

Die PDF-Daten werden bei Nomos (anders als früher üblich war und heute auch noch in vielen Verlagen gehandhabt wird) nicht von den AutorInnen „reprofähig“ angeliefert, sondern in einem aufwendigen Verfahren auf Grundlage der mit einem handelsüblichen Textverarbeitungs-System erstellten Dateien ins XML-Format und dann über XSLT nach XSL-FO umgewandelt, damit am Ende verwertbare strukturierte Daten vorliegen. Der Satz wird nicht in Billiglohnländer ,ausgelagert“, sondern im Haus erledigt, damit die Hersteller die Texte möglichst verstehen und mit den AutorInnen direkt kommunizieren können.

In Zukunft werden auf dieser Grundlage alternativ oder parallel HTML-Fassungen der Texte angeboten werden können und müssen, die 1. strukturiert und daher besser durchsuchbar, 2. in der Anzeige skalierbar und daher besser lesbar und 3. leichter barrierefrei zu gestalten sind - aber derzeit noch mit hohem Aufwand manuell um Seitenzahlen ergänzt werden müssen, um die Zitiersicherheit zu gewährleisten.

30 Damit ist nicht nur die Vergabe der (hierarchisierten) Schlagworte an sich gemeint, sondern auch die weitere Anreicherung um Synonyme, fremdsprachige Entsprechungen etc., um die Werke auch international besser zu verbreiten.

31 Neben den für den Handel üblichen Standard-Klassifikationen (z.B. WGS oder THEMA) müssen z.B. Bibliotheksklassifikationen (BIC, BISAC, RVK, Library of Congress, Dewey Decimal Classification etc.). vergeben werden. 
rien, ${ }^{32}$ Dienstleister ${ }^{33}$ und Suchmaschinen ${ }^{34}$ verteilt werden, damit die Inhalte der Veröffentlichungen noch leichter erschlossen werden können. ${ }^{35}$

Wir glauben, dass gutes Marketing im Bereich wissenschaftliches Publizieren weniger darauf abzielt, ein paar mehr Ausgaben eines Buchs oder einer Zeitschrift zu verkaufen. Vielmehr geht es darum, den Inhalt der Publikationen für die weltweite Wissenschafts-Community und die interessierte Öffentlichkeit sichtbar zu machen.

Verglichen mit diesem Aufwand sind die eigentlichen Kosten für das Drucken und Binden von Büchern und Zeitschriften eher gering. Eine Open Access-Publikation kostet den Verlag daher fast ebenso viel wie eine gedruckte Version des gleichen Inhalts - aber der Umsatz, der durch den Verkauf von Büchern und durch Zeitschriftenabonnements entsteht, nimmt signifikant ab oder (wenn es keine Druckversion mehr gibt) versiegt vollständig. Um die Kosten für eine optimale Verbreitung wissenschaftlicher Veröffentlichungen und der ihnen enthaltenen Forschungsergebnisse zu decken, benötigen Verlage daher Zuschüsse in Form so genannter Book Processing Charges (BPCs).

Immer mehr akademische Einrichtungen und Wissenschaftsorganisationen erkennen die Notwendigkeit einer Finanzierung akademischer Publikationen an. Geldmittel, die bislang für den Erwerb von Büchern und Zeitschriften verwendet wurden, werden nach und nach für die Finanzierung von Open Access-Publikationen umgeschichtet und immer mehr Einrichtungen finanzieren solche Publikationen durch Zuschüsse. Wir unterstützen unsere Autoren dabei, Förderinstitutionen zu finden und beraten sie, welche Veröffentlichungsmethode für ihre Arbeit am geeignetsten ist:

- Welche Art der Veröffentlichung ist am besten für meine Arbeit: ist es empfehlenswert, die Arbeit nur elektronisch zu veröffentlichen oder brauche ich auch eine gedruckte Version?

- Wann sollte meine Arbeit Open Access zur Verfügung gestellt werden?

- Was ist eine Creative Commons-Lizenz und welche dieser Lizenzen sollte ich verwenden?

32 Z.B. DOAB, OAPEN, Open Research Library und eventuell auch Fachrepositorien wie SSRN - hier steckt die Kooperation noch in den Kinderschuhen.

33 Z.B. die stetig wachsende Zahl von Handelspartnern, die sich allmählich auch auf Open Access einstellen (EBSCO, JSTOR, iTunes, Google Play etc.), und natürlich Bibliothekskataloge und -verbünde.

34 Z.B. Google, Google Scholar, Google, Baidu, Scopus Books, Clarivate Book Citation Index.

35 Dabei lassen sich die Kosten pro Werk nur schwer beziffern, da nicht die Lieferung der Daten an sich, Kosten verursacht, sondern eher die Vorbereitung der Daten unter Berücksichtigung der vorgegebenen und teilweise sehr unterschiedlichen Standards, die für die einzelnen Partner gelten. es kommen ständig neue „Ziele“ hinzu. Das ist „Work in Progress“ und wohl kein Verlag kann alle Standards jederzeit pflegen. 


\section{Modell 1: Open Access mit paralleler Druckausgabe (hybrides Modell)}

AutorInnen und HerausgeberInnen der bei Nomos erscheinenden Werke haben die Möglichkeit, zeitgleich mit der Veröffentlichung einer Druckausgabe auch eine elektronische Ausgabe Open Access online verfügbar zu machen (hybride Publikation). ${ }^{36}$ Der Verlag geht davon aus, dass eine solche hybride Publikation jedenfalls bei Monographien zumindest auf absehbare Zeit den Interessen aller Beteiligten am besten entspricht:

- Die hochwertig hergestellte Druckausgabe hat einen eigenen Wert: Sie bietet einen anderen Zugang zu den Inhalten als die elektronische Fassung und ist besser geeignet, Dritte im Wege der persönlichen Ansprache oder durch die Auslage bei Veranstaltungen auf die Arbeit und ihre Ergebnisse aufmerksam zu machen.

- Zudem ist die Druckausgabe problemlos archivierbar und nach vielen Promotionsordnungen erforderlich.

- Und schließlich können die Erlöse aus dem Verkauf der Druckausgabe dazu beitragen, den Zuschussbedarf zu reduzieren.

Wenn wir gedruckten Büchern, insbesondere Monographien, auch im Zeitalter des Open Access eine besondere Bedeutung zumessen, sehen uns hier durch die sogenannte "Stavanger-Erklärung " ${ }^{37}$ vom Oktober 2018 bestätigt:

"Research shows that paper remains the preferred reading medium for longer single texts, especially when reading for deeper comprehension and retention, and that paper best supports long-form reading of informational texts. "

Die "Stavanger-Erklärung" fasst die Ergebnisse einer mehrjährigen Forschungsinitiative zusammen. An dieser Initiative "Evolution of Reading in the Age of Digitisation (E-READ)" waren fast 200 auf den Gebieten des Lesens, des Publizierens und der Lese- und Schreibfähigkeit tätige WissenschaftlerInnen aus ganz Europa beteiligt, die sich mit den Auswirkungen der Digitalisierung auf die Lesepraxis befassen.

Die Open Access-Veröffentlichung erfolgt unter einer so genannten Creative Commons-Lizenz. Wir empfehlen hier eine CC-BY-NC-ND-Lizenz, die unter anderem eine kommerzielle Nutzung durch Dritte und eine Mischung mit anderen Werken ausschließt. Solche Nutzungen bleiben der Zustimmung durch die UrheberInnen vorbehalten. Aber natürlich richten wir uns nach den Wünschen unserer AutorIn-

36 Wenn die Publikation in Zusammenarbeit mit anderen, insbesondere ausländischen Verlagen erfolgt, die ihrerseits für eine bestimmte Region das exklusive Recht zum Vertrieb einer elektronischen Ausgabe des Werkes übertragen bekommen, muss die Möglichkeit einer Open Access-Publikation im Einzelfall geprüft werden. Dasselbe gilt für den Fall, dass die Publikation durch Dritte finanziert wird, die ihrerseits eine unmittelbare Open Access-Publikation parallel zur Veröffentlichung der Druckausgabe ausschließen. Das betrifft etwa Arbeiten, die vom Förderungsfonds Wissenschaft der VG Wort gefördert werden, der sich wiederum aus Einnahmen der VG Wort speist, die bei Open Access wegfallen.

37 http://ereadcost.eu/stavanger-declaration/. 
nen und den Vorgaben eventueller Förderinstitutionen. Die primäre Online-Veröffentlichung erfolgt im Rahmen der Nomos eLibrary.

Das Preismodell differenziert zwischen einer klassischen Publikationskostenbeihilfe und einem Open-Access-Zuschlag der die Mindereinnahmen ausgleichen soll, die wir infolge der Open-Access-Publikation erwarten. Das beruht auf Schätzungen und muss gegebenenfalls in Zukunft an die realen Entwicklungen angepasst werden.

\begin{tabular}{|l|r|r|r|r|r|}
\hline \multicolumn{7}{|c|}{ Angebot für Open Access (hybrid ${ }^{38}$} \\
\hline von-bis Seiten & $\begin{array}{c}\text { Pub.- } \\
\text { Beihilfe } \\
\text { Druck- } \\
\text { ausgabe }\end{array}$ & $\begin{array}{c}\text { OA- } \\
\text { Zuschlag } \\
\text { netto }\end{array}$ & $\begin{array}{c}\text { Summe } \\
19 \% \text { Ust. }\end{array}$ & $\begin{array}{c}\text { Summe } \\
\text { brutto }\end{array}$ \\
\hline $0-200$ & $2.268,66 €$ & $1.531,34 €$ & $3.800,00 €$ & $722,00 €$ & $4.522,00 €$ \\
\hline $200-300$ & $3.343,28 €$ & $2.256,72 €$ & $5.600,00 €$ & $1.064,00 €$ & $6.664,00 €$ \\
\hline $300-400$ & $3.940,60 €$ & $2.659,90 €$ & $6.600,50 €$ & $1.254,10 €$ & $7.854,60 €$ \\
\hline $400-500$ & $4.477,61 €$ & $3.022,39 €$ & $7.500,00 €$ & $1.425,00 €$ & $8.925,00 €$ \\
\hline $500-600$ & $4.895,52 €$ & $3.304,48 €$ & $8.200,00 €$ & $1.558,00 €$ & $9.758,00 €$ \\
\hline $600-700$ & $5.134,33 €$ & $3.465,67 €$ & $8.600,00 €$ & $1.634,00 €$ & $10.234,00 €$ \\
\hline jede weitere & $2,23 €$ & $1,50 €$ & $3,73 €$ & $0,71 €$ & $4,44 €$ \\
Seite & & & & & \\
\hline
\end{tabular}

Für bestimmte Personengruppen gelten besondere Konditionen. Das betrifft unter anderem die Angehörigen öffentlich-geförderter wissenschaftlicher Einrichtungen in Deutschland ${ }^{39}$ oder Personen, bei denen die Max-Planck-Digital Library die Open Access-Ausgabe finanziert. ${ }^{40}$

\section{Modell 2: Ausschließliche Online-Veröffentlichung mit Open Access (eOnly-Modell)}

Neben dem hybriden Modell besteht für AutorInnen und HerausgeberInnen auch die Möglichkeit, Ihr Werk ausschließlich online und Open Access zu veröffentlichen, also ohne parallele Druckausgabe. Hier ist zu beachten, dass der Verlag keine Vertriebserlöse mehr erzielen kann, so dass der Zuschuss den gesamten Aufwand für die Herstellung und die Verbreitung des Werkes abdecken muss. Zwar

38 Der Aufwand für eine besondere Ausstattung der Druckausgabe, die Herstellung einer bestimmten Mindestzahl von Exemplaren dieser Druckausgabe oder aufwendige Satzarbeiten kann gegebenenfalls gesondert abgerechnet werden. Unter bestimmten Umständen sind Rabatte möglich, die im Einzelfall ausgehandelt werden.

39 Hier gewähren wir aufgrund der mit der DFG vereinbarten Allianz-Lizenz für die Nomos eLibrary einen Nachlass von ca. $10 \%$ auf die in den Tabellen genannten Beträge.

40 Im Rahmen einer Kooperation mit der Max-Planck-Digital Library (MPDL) kommt für Arbeiten aus dem Umfeld von Max-Planck-Instituten, die sich auch an den regulären Publikationskostenbeihilfen beteiligen eine Pauschalierung dieser Beihilfen unabhängig vom Umfang in Betracht. Die MPDL übernimmt dann den OA-Aufschlag. Das entsprechende Modellprojekt läuft seit 2017 und noch mindestens bis 2020 . 
spart der Verlag einen Teil der Druckkosten ein, wobei wir davon ausgehen, dass es im Interesse der AutorInnen und HerausgeberInnen liegt, wenn wir etwa für die Auslage bei Veranstaltungen, aber auch für die Ansprache potenzieller Rezensenten zumindest eine kleine Druckauflage herstellen. Die Einsparungen bei den Druckkosten gleichen jedoch die zu erwartenden Rückgänge bei den Vertriebserlösen nicht aus. Im Ergebnis ist der Zuschussbedarf daher höher als im hybriden Modell.

Die Arbeiten werden in diesem Modell in der Regel unter einer Creative Commons Attribution Lizenz (CC-BY Lizenz) in der aktuellen Version veröffentlicht. Es steht den AutorInnen jedoch frei, eine andere CC-Lizenz zu wählen.

\begin{tabular}{|l|r|r|r|}
\hline \multicolumn{4}{|c|}{ Angebot für Open Access (eOnly) } \\
\hline \multicolumn{1}{|c|}{ von-bis Seiten } & Summe netto & \multicolumn{1}{c|}{$\mathbf{1 9 \% \text { Ust. }}$} & \multicolumn{1}{c|}{ Summe Brutto } \\
\hline $0-200$ & $4.600,00 €$ & $874,00 €$ & $5.474,00 €$ \\
\hline $200-300$ & $6.900,00 €$ & $1.311,00 €$ & $8.211,00 €$ \\
\hline $300-400$ & $8.000,00 €$ & $1.520,00 €$ & $9.520,00 €$ \\
\hline $400-500$ & $9.200,00 €$ & $1.748,00 €$ & $10.948,00 €$ \\
\hline $500-600$ & $10.100,00 €$ & $1.919,00 €$ & $12.019,00 €$ \\
\hline $600-700$ & $10.600,00 €$ & $2.014,00 €$ & $12.614,00 €$ \\
\hline jede weitere Seite & $4,00 €$ & $0,76 €$ & $4,76 €$ \\
\hline
\end{tabular}

\section{Schluss}

Das Fallbeispiel Nomos illustriert zum einen, dass Open Access kein Mittel ist, die Kosten wissenschaftlicher Publikationen zu senken - auch wenn es in der Diskussion immer wieder Stimmen gibt, die diese Hoffnung zum Ausdruck bringen und obwohl es einige Verlage gibt, die ihre AutorInnen im Glauben wiegen, dass ihre Erkenntnisse von alleine zum potentiellen Leser finden werden. Die möglichst zielgenaue Verbreitung wissenschaftlicher Erkenntnisse sollte aber auch nicht den WissenschaftlerInnen selbst zugemutet werden. Vielmehr gibt es dafür eine bewährte Infrastruktur aus Bibliotheken und Verlagen, die auf der einen Seite dabei hilft, aus der Fülle von Publikationen möglichst diejenigen zu finden, die zur eigenen Forschungsfrage etwas beizutragen haben - und auf der anderen Seite maßgeblich dazu beträgt, dass Erkenntnisse nicht einfach wieder verloren gehen, weil ihr Nutzen nicht unmittelbar auf der Hand liegt. Auch und gerade wenn man mit der Stavanger-Erklärung davon ausgeht, dass umfangreiche Texte, die in die Tiefe gehen, am besten über gedruckte Bücher wahrgenommen und verarbeitet werden können, ermöglicht die sinnvolle Aufbereitung elektronischer Veröffentlichung einen sehr viel einfacheren Zugang zu diesen Texten. Exzellente Wissenschaft sollte nicht hinter verschlossenen Türen bleiben - und muss es auch nicht. 